\title{
Synthesis of-4-((4-trimethylsilyl-1H-1,2,3-triazol-1- yl)methyl)-2H-chromen-2-ones: A novel class of heteroaryl anionic synthon
}

\section{Sowmiya Carmel Y, Noor Shahina Begum, Prasad N L \& Hari Prasad Suresh}

To cite this article: Sowmiya Carmel Y, Noor Shahina Begum, Prasad N L \& Hari Prasad Suresh (2018) Synthesis of-4-((4-trimethylsilyl-1H-1,2,3-triazol-1-yl)methyl)-2H-chromen-2-ones: A novel class of heteroaryl anionic synthon, Synthetic Communications, 48:5, 588-593, DOI: 10.1080/00397911.2017.1416634

To link to this article: https://doi.org/10.1080/00397911.2017.1416634

View supplementary material ¿

央 Published online: 25 Jan 2018.

6 Submit your article to this journal

III Article views: 130

View Crossmark data $[\pi$ 


\title{
Synthesis of-4-((4-trimethylsilyl-1H-1,2,3-triazol-1-yl)methyl)- $2 \mathrm{H}$-chromen-2-ones: A novel class of heteroaryl anionic synthon
}

\author{
Sowmiya Carmel Y, Noor Shahina Begum, Prasad N L, and Hari Prasad Suresh
}

Department of Post-Graduate Studies in Chemistry, Bangalore University, Bangalore, India

\begin{abstract}
A one-pot synthesis of some novel anionic scaffolds: the substituted-4((4-trimethylsilyl-1H-1,2,3-triazol-1-yl)methyl)-2H-chromen-2-one is reported. Reaction of 10 different substituted bromomethylcoumarins with trimethylsilylacetylene and sodium azide in the presence of copper(I) iodide catalyst gave the corresponding heteroaryl conjugates: the substituted-4-((4-trimethylsilyl-1H-1,2,3-triazol-1-yl)methyl)$2 \mathrm{H}$-chromen-2-one in $70-92 \%$ yields. The structures of the synthesized compounds have been completely characterized by spectral and elemental analyses. For the first time, the representative single-crystal X-ray structure analysis of 6-methoxy-4-((4-trimethylsilyl- $1 \mathrm{H}-1,2$, 3-triazol-1-yl)methyl)-2H-chromen-2-one is reported which confirms the formation of anionic synthon which bears the trimethylsilyl-group.
\end{abstract}

\section{GRAPHICAL ABSTRACT}

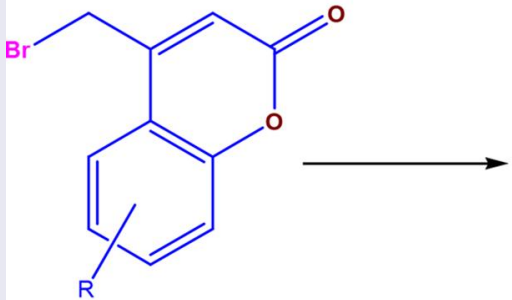

1a-j

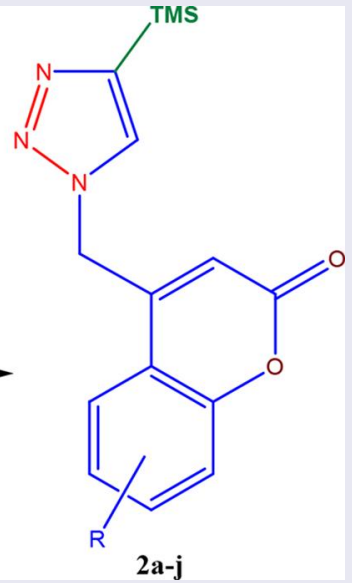

\section{ARTICLE HISTORY}

Received 31 July 2017

\section{KEYWORDS}

Anionic synthons; conjugate molecules; coumarins; heterocyclic scaffolds; triazoles

CONTACT Hari Prasad Suresh hariprasad@bub.ernet.in Department of Post-Graduate Studies in Chemistry, Central College Campus, Bangalore University, Bangalore 560001, India.

Color versions of one or more of the figures in the article can be found online at www.tandfonline.com/lsyc. (4) Supplemental data (full experimental detail, IR, ${ }^{1} \mathrm{H}-\mathrm{NMR},{ }^{13} \mathrm{C}-\mathrm{NMR}$, mass spectra, and microanalysis) can be accessed on the publisher's website. 


\section{Introduction}

The concept of hybrid pharmacophore approach has led to a detailed study of possible biosynergizing of some pharmacologically important heterocyclic compound conjugates. It is well established that a combination of diverse heterocyclic cores in one molecule often leads to new pharmacological profiles with increased biological activity and reduced toxicity of the newly synthesized hybrid molecules. ${ }^{[1]}$

Literature survey reveals that coumarinyl backbone in assimilation with some nitrogen-containing heterocyclic moieties such as triazoles significantly increases the antimicrobial activity and broadens their antimicrobial spectrum. In recent years, a library of coumarin derivatives conjugated with 1,2,3-triazole has gained paramount importance in various fields of bioactivity and has also evoked much attention in forming scaffolds in numerous synthetic methodologies. ${ }^{[2-4]}$ However, to our knowledge, most of the reported conjugate molecules have not been converted into active pharmacodynamic agents.

The 1,2,3-triazole moiety by itself is a versatile heterocycle with a diverse range of biological activities. ${ }^{[5]}$ In this regard, the substitution of trimethylsilyl group at position 4 of the triazoyl moiety would lead to the formation of novel anionic synthons. The synthons are further regiospecifically transformed to form larger molecules with a wide range of chemical and pharmacological properties. The transformations would exploit the $\alpha$ - and $\beta$-silyl effects.

One of the important routes for the preparation of triazole moieties is using Sharpless click chemistry. ${ }^{[6]}$ The methodology uses 1,3-dipolar cycloaddition of alkynes and azides to form $\mathrm{N}_{1}$-substituted 1,2,3-triazoles in the presence of a $\mathrm{Cu}(\mathrm{I})$ catalyst. ${ }^{\text {[7] }}$

Our laboratory deals with the synthesis and reactions of organosilyl-based reagents. ${ }^{[8]}$ In this piece of work, we report for the first instance of time, the synthesis of some novel conjugate heterocyclic scaffolds: the substituted-4-((4-trimethylsilyl-1H-1,2,3-triazol-1-yl) methyl)-2H-chromen-2-ones. The molecules, we expect, would serve as precursors for

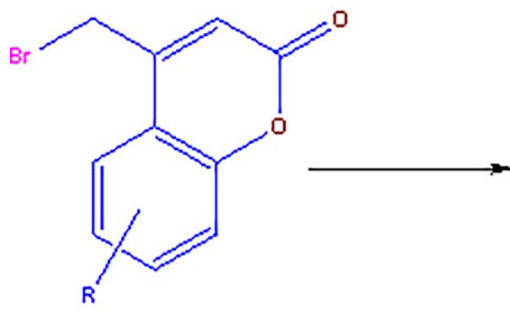

$\mathbf{l a - j}$

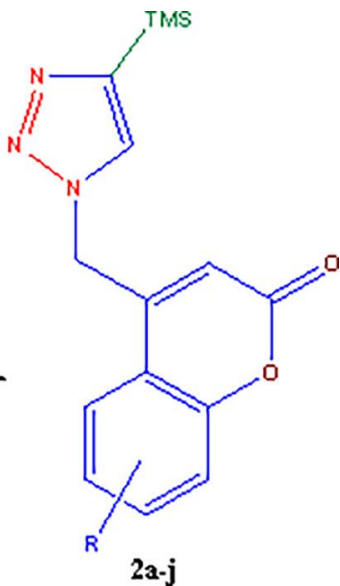

$\mathbf{2 a - j}$

Scheme 1. Reagents and conditions: $1 \mathrm{a}-\mathrm{j}(2 \mathrm{mmol})$; trimethylsilylacetylene $(2 \mathrm{mmol})$; sodium azide $(1.5 \mathrm{mmol}) ;$ and Cul $(1 \mu \mathrm{mol})-10 \mathrm{~mL}$ acetone/water $(1: 1), 0-28^{\circ} \mathrm{C}$, stirring $8 \mathrm{~h}$. 


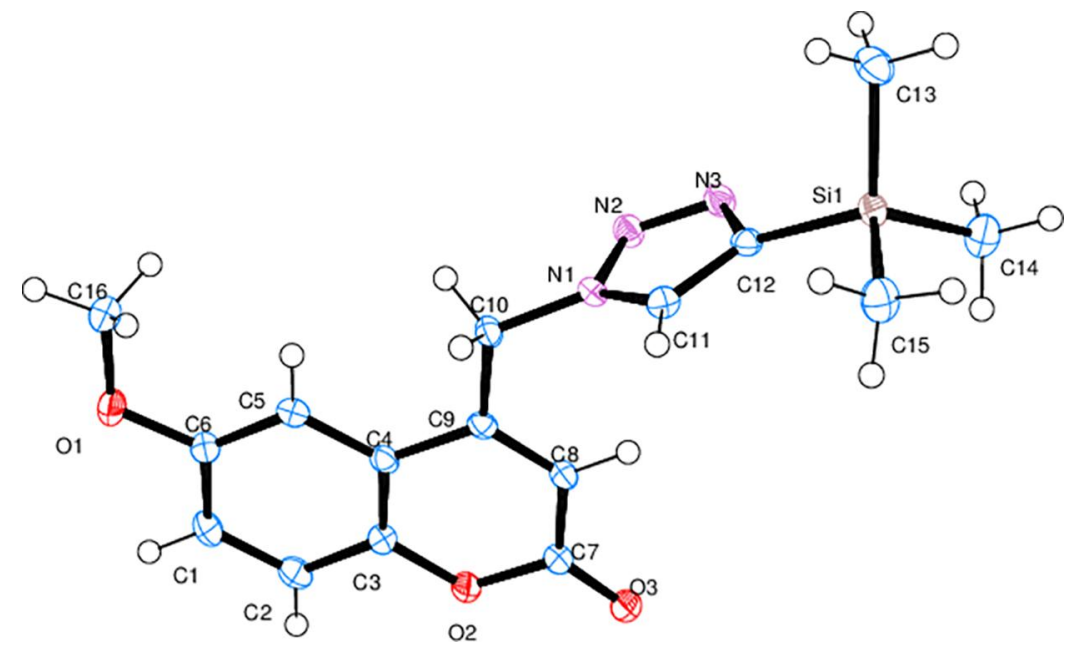

Figure 1. ORTEP view of 6-methoxy-4-((4-trimethylsilyl-1H-1,2,3-triazol-1-yl)methyl)-2H-chromen-2one (2a).

the synthesis of larger molecules with a wide range of chemical and pharmocodynamic properties. $^{[9]}$

The one-pot reaction of 4-bromomethylcoumarins $\mathbf{1 a}-\mathbf{j}$ with trimethylsilylacetylene (TMSA) and sodium azide in the presence of CuI catalyst in acetone/water solvent system over $8 \mathrm{~h}$ yielded the novel anionic synthons: the substituted-4-((4-trimethylsilyl- $1 H-1,2$, 3-triazol-1-yl)methyl)-2H-chromen-2-one. 2a-j in 70-92\% yields (Scheme 1).

\section{Results and discussion}

The 4-bromomethylcoumarins 1a-j were synthesized using Pechmann cyclization of substituted phenols with 4-bromoethylacetoacetate in the presence of concentrated $\mathrm{H}_{2} \mathrm{SO}_{4}$ as a dehydrating agent. ${ }^{[10]}$

Individually, stirring a mixture of 4-bromomethylcoumarins (1a-j), sodium azide, TMSA, and copper(I) iodide catalyst in $1: 1$ acetone/water at $0{ }^{\circ} \mathrm{C}$ to ambient temperature over $8 \mathrm{~h}$ yielded the trimethylsilyl-substituted triazolylcoumarins $\mathbf{2 a - j}$ conjugates in $70-92 \%$ isolated yields as depicted in Scheme 1. The optimum yields of the products are given in Table 1.

In this regard, we now wish to report for the first instance of time, the single-crystal $\mathrm{XRD}$ of the conjugate anionic synthon: the trimethylsilyl-bearing coumarinyltriazolyl compound 2a. We were able to isolate $\mathbf{2 a}$ as good single crystals by slow evaporation technique for X-ray structure analysis. The ORTEP view of 2a is given in Figure 1.

To our knowledge, there exist no reports for the single-crystal XRD of such conjugate anionic synthons bearing the trimethylsilyl group. Therefore, we now report for the first instance of time the trimethylsilyl-bearing multifunctional heterocycle conjugate molecule. The complete details of XRD characterization is provided in supplementary material. ${ }^{[1-17]}$

The possible mechanism for formation of products $\mathbf{2 a - j}$ is reasoned to go through the cycle as highlighted in Scheme 2. The mechanism follows the path as given by Worell et al. ${ }^{[18]}$ 
Table 1. Synthesis of-4-((4-trimethylsilyl-1H-1,2,3-triazol-1-yl)methyl)-2H-chromen-2-ones 2a-j.

Entry Reactant

$1 \mathbf{b}$

C

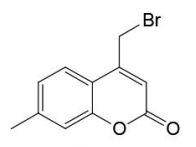

1c

d

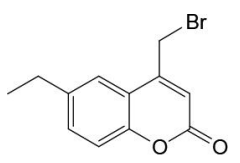

1 d

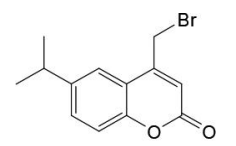

$1 e$

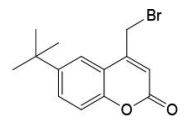

$1 f$

g

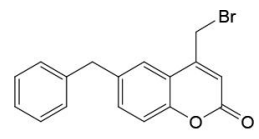

$1 g$

h

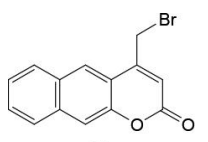

$1 \mathrm{~h}$

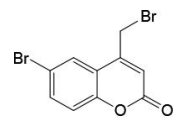

1 i

\section{j}

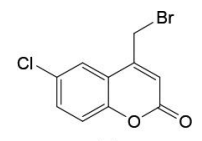

1j

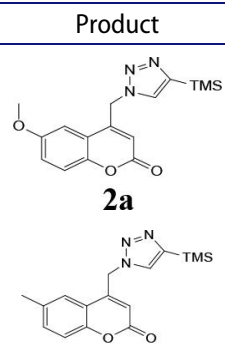

$2 b$
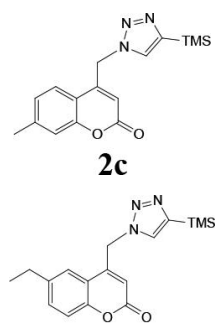

2d

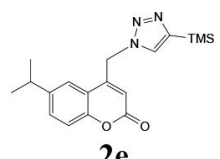

2 e
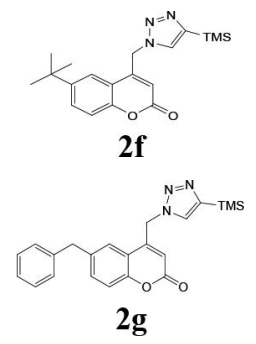

97

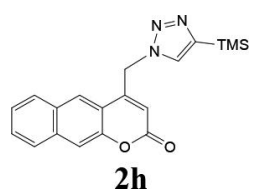

91

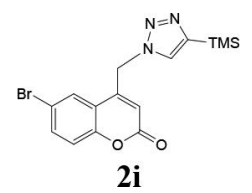

95

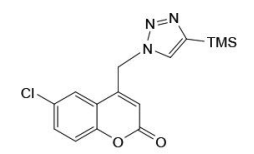

$2 \mathrm{j}$ 


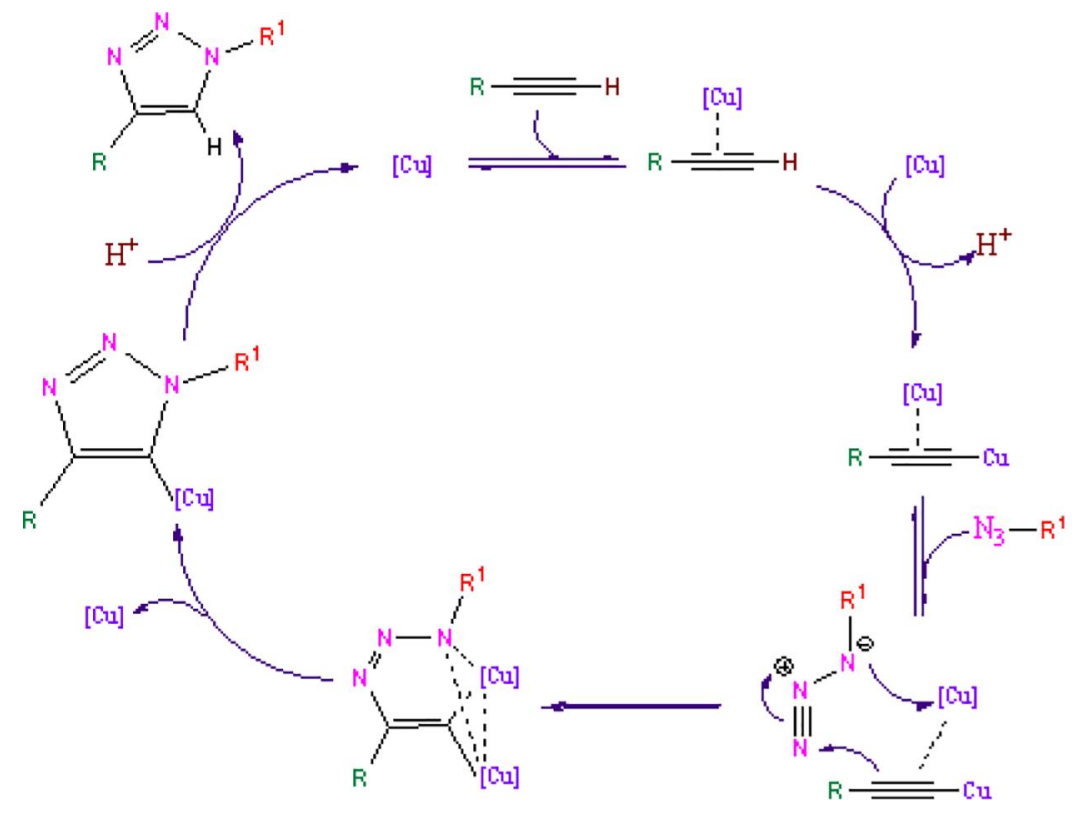

Scheme 2. Mechanism for the formation of products $2 a-j$.

\section{Experimental section}

\section{General procedure for the preparation of substituted-4-((4-trimethylsilyl-1H- 1,2,3-triazol-1-yl)methyl)-2H-chromen-2-one (2a-j)}

To an ice-cold suspension of differently substituted bromomethyl coumarins $\mathbf{1 a}-\mathbf{j}$ $(2 \mathrm{mmol})$, sodium azide $(1.5 \mathrm{mmol})$, and copper iodide $(1 \mu \mathrm{mol})$ in $10 \mathrm{~mL}(1: 1)$ of aqueous acetone was added dropwise trimethylsilyl acetylene $(2 \mathrm{mmol})$ over a period of $30 \mathrm{~min}$. The resultant mixture was allowed to attain room temperature and further stirred for a period of $8 \mathrm{~h}$. The reactions were monitored by TLC and GC through microworkup of aliquots. After the completion of reaction as indicated by the chromatograms, excess acetone was removed under rotary evaporation and crude product was purified by column chromatography, using silica gel (100-200 mesh) and 2:5 ethyl acetate-petroleum benzine $\left(60-74^{\circ} \mathrm{C}\right.$ fraction) to obtain compounds $2 \mathbf{2}-\mathbf{j}$. The isolated yields are reported in Table 1 . The representative spectral details for $\mathbf{2 a}$ is given in the following, and the single-crystal XRD details are given in the supplementary material.

6-Methoxy-4-((4-trimethylsilyl-1H-1,2,3-triazol-1-yl)methyl)-2H-chromen-2-one (2a): The product was obtained as yellow solid (87\%); Melting point: $180-184^{\circ} \mathrm{C}$; IR $\left(\mathrm{cm}^{-1}\right)$ : 3130, 2968, 2851, 1713, 1574, 1493, 1423, 1348, 1244, 1163, 1107, 1028, 941,841, 762, 631, 575, 511; ${ }^{1} \mathbf{H}$ NMR: (400 MHz, $\left.\mathrm{CDCl}_{3}, \delta / \mathrm{ppm}\right) 0.33$ (s, $\left.9 \mathrm{H}\right), 3.82$ (s, 3H), 5.72 (s, 2 $\mathrm{H}), 6.07(\mathrm{~s}, 1 \mathrm{H}), 7.08(\mathrm{~d}, J=2.8 \mathrm{~Hz}, 1 \mathrm{H}), 7.15(\mathrm{dd}, J=6.4 \mathrm{~Hz}, 1 \mathrm{H}), 7.31(\mathrm{~d}, J=9.2 \mathrm{~Hz}$, $1 \mathrm{H}), 7.57$ (s, $1 \mathrm{H}) ;{ }^{13} \mathrm{C}$ NMR: (100 MHz, $\left.\mathrm{CDCl}_{3}, \delta / \mathrm{ppm}\right):-1.0,49.9,56.1,106.3,115.9$, $117.5,118.6,120.3,129.4,148.0,148.3,156.4,160.2$; CHNS: Calculated C: $58.34 \%, \mathrm{H}$ : $5.81 \%, \mathrm{~N}: 12.76 \%$; Found C: $58.28 \%, \mathrm{H}: 5.80 \%, \mathrm{~N}: 12.72 \%$. 


\section{Acknowledgment}

Grateful thanks are due to the (i) Bangalore University, (ii) University Grants Commission, Government of India, and (iii) Department of Science and Technology, Government of India, New Delhi for financial assistance. SCY thanks the St. Joseph's Pre-University College and the organizers of the International Conference on Science and Technology: Future Challenges on Science and Solutions (STFCS-2016) held at Mysore on 8th and 9th August 2016, under the auspices of Indian Japan Promotion of Sciences Alumni Association, University of Mysore, and Japan Science Promoting Society for awarding the best poster award entitled "Synthesis of some 4-trimethylsily1,2,3-triazolecoumarins". PNL thanks the University Grants Commission, Government of India, New Delhi, for a Junior Research Fellowship. We are also thankful to the NMR Department, Indian Institute of Science-Bangalore and Vellore Institute of Technology, Vellore for spectral analysis.

\section{Funding}

This work was supported by the University Grants Commission, Govt. of India, New Delhi [Grant Number. 36-42/2008 (SR) UGC, Dated 26.10.2017].

\section{References}

[1] (a) Solomon, V. R.; Hu, C.; Lee, H. Bioorganic Med. Chem. 2010, 18, 1563-1572. DOI: 10.1016/ j.bmc.2009.08.068; (b) Martins, P.; Jesus, J.; Santos, S.; Raposal, L. R.; Rona-Rodrigues, C.; Baptista, P. V.; Fernandes, A. R. Molecules 2015, 20, 16852-16891. DOI: 10.3390/ molecules200916852.

[2] Tripathi, R. P.; Yadav, A. K.; Ajay, A.; Bisht, S. S.; Chaturvedi, V.; Sinha, S. K. Eur. J. Med. Chem. 2010, 45, 142-148. DOI: 10.1016/j.ejmech.2009.09.036.

[3] Sreedhar, B.; Reddy, P. S.; Kumar, N. S. Tetrahedron Lett. 2006, 47, 3055-3058. DOI: 10.1016/j. tetlet.2006.03.007.

[4] Naik, R. J.; Kulkarni, M. V.; Sreedhar Ranganath Pai, K.; Nayak, Pawan. G. Chem. Biol. Drug. Res. 2012, 80, 516-523. DOI: 10.1111/j.1747-0285.2012.01441.x.

[5] (a) Shafran, E. A.; Bakulev, V. A.; Rozin, Y. A.; Shafran, Y. M. Chem. Het. Comp. 2008, 44(9), 1040-1069. DOI: 10.1007/s10593-008-0155-9; (b) Huo, J.; Hu, H.; Zhang, M.; Hu, X.; Chen, M.; Chen, D.; Liu, J.; Xiao, G.; Wang, Y.; Wen, Z. RSC Adv. 2017, 7, 2281-2287. DOI: 10.1039/ c6ra27012c.

[6] Kolb, Hartmuth C.; Finn, M. G.; Barry Sharpless, K. Angew. Chem. Int. Ed. 2001, 40, 2004-2021. DOI: 10.1002/1521-3773(20010601).

[7] Pasini, D. Molecules 2013, 18, 9512-9530. DOI: 10.3390/molecules18089512.

[8] See Jeevan, C. A. S.; Krishnamurthy, M. S.; Begum, N. S.; HariPrasad, S. Tetrahedron Lett. 2016, 57, 3231-3234. DOI: 10.1016/j.tetlet.2016.06.051.

[9] Daria, Y. D.; Andrei, V. B. Beilstein J. Org. Chem. 2016, 12, 874-881. DOI: 10.3762/bjoc.12.86.

[10] Raviraj, A. K.; Kulkarni, M. V. Asian J. Chem. 2014, 26, 1077-1080. DOI: 10.14233/ ajchem.2014.15865.

[11] Bruker, SMART, SAINT-Plus, SADABS. Bruker Axs Inc: Madison, Wisconcin, USA, 1998.

[12] Sheldrick, G. M. Acta Cryst. A 2008, 64, 112. DOI: 10.1107/S0108767307043930.

[13] Nardelli, M. Acta Cryst. C 1983, 39, 1141. DOI: 10.1107/S0108270183007696.

[14] Farrugia, L. J. J. Appl. Cryst. 1997, 30, 565. DOI: 10.1107/S0021889897003117.

[15] http://www.ccdc.cam.ac.uk/mercury.

[16] Watkin, D. J.; Prout, C. K.; Pearce, L. J. CAMERON; Chemical Crystallography Laboratory, University of Oxford: England, 1996.

[17] Brandenburg, K.; Putz, H. Crystal Impact. GbR, Bonn, Germany, 2005.

[18] Worell, B. T.; Malik, J. A.; Fokin, W. Science 2013, 340, 457-460. DOI: 10.1126/ science.1229506. 